\title{
L'impact de l'utilisation du Portfolio européen des langues en cycle de Licence de Sciences économiques
}

\section{Anne-Marie Barrault-Méthy}

\section{(2) OpenEdition}

\section{Journals}

Édition électronique

URL : http://journals.openedition.org/asp/806

DOI : $10.4000 /$ asp. 806

ISBN : 978-2-8218-0400-5

ISSN : 2108-6354

\section{Éditeur}

Groupe d'étude et de recherche en anglais de spécialité

\section{Édition imprimée}

Date de publication : 1 janvier 2005

Pagination : 103-120

ISSN : 1246-8185

\section{Référence électronique}

Anne-Marie Barrault-Méthy, "L'impact de l'utilisation du Portfolio européen des langues en cycle de Licence de Sciences économiques », ASp [En ligne], 47-48 | 2005, mis en ligne le 16 février 2010, consulté le 19 avril 2019. URL : http://journals.openedition.org/asp/806 ; DOI : 10.4000/asp.806

Ce document a été généré automatiquement le 19 avril 2019

Tous droits réservés 


\title{
L'impact de l'utilisation du Portfolio européen des langues en cycle de Licence de Sciences économiques
}

\author{
Anne-Marie Barrault-Méthy
}

1 Le Portfolio européen des langues (PEL), développé entre 1998 et 2000, est présenté par le Conseil de l'Europe comme « un document dans lequel toute personne qui apprend ou a appris une langue - que ce soit à l'école ou en dehors - peut consigner ses connaissances linguistiques et ses expériences culturelles, ce qui peut l'inciter à réfléchir sur son apprentissage $»^{1}$. Le PEL se compose de trois parties principales : le Passeport, qui donne une vue d'ensemble des compétences en langues de son propriétaire, la Biographie langagière, qui vise à favoriser la réflexion sur l'apprentissage des langues, et le Dossier, qui illustre les compétences en rassemblant les productions langagières les plus significatives. À ces trois parties s'ajoute une Annexe qui décrit les compétences en fonction du système des niveaux du Cadre européen commun de référence pour les langues.

2 Le PEL bénéficie de l'enthousiasme de nombre d'enseignants, même si beaucoup hésitent encore à franchir le pas et à l'utiliser avec leurs étudiants. Plusieurs raisons expliquent cet accueil favorable :

- le PEL jouit d'une reconnaissance internationale ;

- il favorise l'auto-évaluation selon une échelle européenne, valable pour toutes les langues ;

- il permet aux étudiants de consigner leur niveau dans toutes les langues qu'ils possèdent à des degrés divers ;

- il stimule la réflexion sur la manière d'apprendre les langues ;

- il propose des objectifs d'apprentissage définis ;

- il peut être mis à jour au fur et à mesure de l'apprentissage ;

- il rend concret l'enseignement des langues à l'université et permet aux candidats à un poste de présenter aux entreprises leurs capacités et leurs réalisations ;

- son coût de duplication, même en version papier et même dans le cadre d'un enseignement de masse, reste raisonnable et sera même rendu nul par les portfolios numériques qui sont sans doute à venir ${ }^{2}$. 
Pour les enseignants de langues, le PEL aide à structurer les enseignements et apporte une reconnaissance institutionnelle et internationale à leur travail. Il relativise l'importance à accorder aux certifications de type TOEFL et TOEIC. Dans le cadre d'une approche cognitive de l'enseignement, dans la mesure où il présuppose que la réflexion sur l'apprentissage favorisera l'apprentissage et développera la motivation, il est l'outil métacognitif par excellence. Pour Schneider et Lenz (2001), le projet du Portfolio possède deux objectifs : fournir, d'une part, un bilan des capacités en langues vivantes et, d'autre part, motiver les apprenants. Si les institutions ont validé le PEL étudiant, le considérant comme une avancée, comment ce dernier est-il perçu par les étudiants auxquels il est destiné ? Suscite-t-il le même enthousiasme ? Est-il motivant? Les conduit-ils à travailler différemment ? Recourent-ils davantage aux méthodes en libre accès dans les centres de langues? Se donnent-ils des objectifs d'apprentissage? Nous proposons, dans ce travail, une étude quantitative permettant de jauger les changements, notamment sur la motivation, induits par une utilisation du PEL qui s'appuie essentiellement sur la Biographie et dans le contexte de l'enseignement présentiel en $1^{\mathrm{e}}$ et $2^{\mathrm{e}}$ années de Sciences économiques. Pour ce faire, nous avons mis en place un protocole d'expérimentation.

\section{Méthode}

\subsection{L'orientation de l'utilisation du PEL}

Le PEL se prête à de multiples utilisations. Little et Perclová (2001) en présentent deux : la première consiste à commencer par le Dossier pour ensuite se fixer des objectifs; la seconde propose de considérer le Dossier comme un élément dynamique qui peut être mis à jour régulièrement et donner lieu à une auto-évaluation de l'apprenant, qui se fixera alors nouveaux objectifs. Pour Kohonen (2001), il en existe une troisième: le Dossier peut constituer l'élément central du dispositif, la Biographie langagière n'intervenant que pour réaliser un bilan en fin de parcours. Nous avons choisi de proposer aux étudiants de s'auto-évaluer au moyen du Portfolio et de Dialang ${ }^{3}$, test diagnostique en ligne permettant l'évaluation en quatorze langues à partir des niveaux du Cadre européen commun de référence. Ensuite, les étudiants étaient invités à se fixer des objectifs d'apprentissage.

5 Plusieurs raisons ont présidé au choix de cette utilisation du PEL. D'une part, l'autoévaluation initiale au moyen de l'Annexe et de la Biographie tient compte de compétences de communication interculturelle qui sont à la fois un préalable et un objectif de l'apprentissage, considéré dans une perspective holistique. D'autre part, nous avons voulu nous appuyer sur les conditions locales, à savoir le multiculturalisme des étudiants, dont un quart sont ressortissants d'un pays étranger, en Licence $1^{\mathrm{e}}$ et $2^{\mathrm{e}}$ années dans un contexte national uniculturel où tous les cours sont dispensés en français (hors cours de langues). Ces conditions diffèrent, par exemple, de celles d'autres pays du Conseil de l'Europe où l'on utilise le Portfolio, par exemple en Suisse, qui compte trois langues officielles. Enfin, orienter le travail sur la Biographie et le Dossier suppose aussi la motivation et l'implication des collègues qui, dans ce cas précis, ont distribué le PEL, mais pas véritablement choisi de l'utiliser. Cette question de la motivation est capitale dans la manière, ou la méthode, de mettre en œuvre le PEL et dans toute l'expérimentation sur ce sujet, comme nous le verrons ci-après. 


\subsection{PEL et motivation}

6 Motiver les apprenants est l'un des deux objectifs qu'assigne au PEL le Comité de l'éducation du Conseil de l'Europe dans ses Principes et lignes directrices. Cependant, dans les faits, le PEL est-il véritablement motivant? Dans son rapport sur la phase pilote de l'utilisation du PEL, Schärer ne répond pas clairement à cette question :

Les enfants [les participants], en effet, ont reconnu que le PEL les encourageait à réfléchir à leur apprentissage et $85 \%$ d'entre eux ont considéré qu'il accroissait leur motivation mais $33 \%$ d'entre eux seulement ont déclaré que le PEL les incitait à participer de manière plus active au processus d'apprentissage linguistique. (Schärer 2000 : 39)

7 Or la motivation est précisément cette dynamique (Gardner \& MacIntyre 1993) évoquée par Schärer. Ce dernier admet d'ailleurs que «la question de la motivation devra faire l'objet de nouvelles réflexions ». Une ambiguïé semblable existe concernant le bilan de l'utilisation du PEL par des apprenants étudiants :

En ce qui concerne le transfert des capacités d'étude, $40 \%$ de ces mêmes étudiants ont indiqué qu'ils étaient prêts à entreprendre des activités comparables d'autoévaluation dans d'autres parties de leur cursus universitaire. Les capacités d'étude attribuées par les étudiants au rôle central du PEL dans leurs modules de langues vivantes comprennent en particulier: la définition d'objectifs d'apprentissage, l'établissement d'un calendrier d'étude, l'accroissement de la motivation, une meilleure compréhension du processus d'apprentissage et une plus grande capacité à la réflexion. (Schärer $2000: 54$ )

8 Ces propos ne permettent pas de connaître le pourcentage des étudiants qui ont jugé le PEL motivant pour apprendre les langues. Tout juste laissent-ils entendre en creux que $60 \%$ ne sont pas prêts à transférer à d'autres matières leur capacité à l'étude acquise grâce au PEL. Pourtant, le rapport de synthèse 2001-2004 (Schärer 2004 : 5) indiquait que " [d]ans la plupart des cas, on a régulièrement constaté des effets positifs sur le processus d'apprentissage, les résultats de l'apprentissage et la motivation de l'apprenant ». Plus loin dans ce même rapport, on trouve une affirmation semblable, qui n'est toujours pas étayée : « [le PEL] influence de manière positive la motivation, le processus et les résultats de l'apprentissage » (Schärer 2004 : 17). Le fait que le PEL renforce la motivation constitue donc pour ses promoteurs plus une hypothèse de travail qu'une affirmation (Little \& Perclová 2001).

9 À supposer que le PEL soit effectivement motivant, comment cette motivation se développe-t-elle ? Selon Little et Perclová,

le défi est donc de faire dire à l'apprenant ce qu'il trouve intéressant et de lui faire partager la responsabilité d'assurer que ce qui se passe dans la classe correspond à ses intérêts et lui est utile. (2001: 41)

10 Pour motiver les étudiants au mieux, il convient d'abord de les interroger sur ce qu'ils trouvent intéressant et ce qu'ils désirent faire, et ensuite de s'assurer que ce qui se passe en cours correspond bien à leurs attentes. Le PEL est conçu comme support à une discussion en TD et comme livre de bord de l'apprentissage. Il doit permettre d'instaurer une dynamique au sein du groupe : en étant conscient de ses processus d'apprentissage, on apprend mieux, ce qui renforce la motivation, laquelle conduit à travailler davantage et plus efficacement, etc. L'instauration de ce cercle vertueux dépend étroitement de l'attitude de l'enseignant à l'égard du PEL. Dans le Guide à l'usage des enseignants et formateurs d'enseignants (Little \& Perclová 2001: 47), les auteurs fournissent aux 
enseignants les explications suivantes: "[...] il existe un parallèle clair entre (i) la détention du PEL par les apprenants et (ii) leur participation au travail et votre appropriation de l'approche de l'enseignement des langues décrite dans ce livre et votre engagement envers celui-ci ». Les conditions locales nous ont empêchée d'utiliser le PEL exactement de cette façon.

\subsection{Le protocole}

Il est résumé au tableau 1.

Tableau 1. Description du protocole

\begin{tabular}{|c|c|}
\hline Description & Commentaires \\
\hline Public cible & $\begin{array}{l}\text { Étudiants de Sciences économiques ayant choisi } \\
\text { l'anglais }\end{array}$ \\
\hline Nombre d'étudiants concernés & 314 en $1^{\mathrm{e}}$ année, 155 en $2^{\mathrm{e}}$ année \\
\hline Nombre d'heures de langue & $\begin{array}{l}1^{\mathrm{e}} \text { année }: 15 \text { heures au } 1^{\mathrm{e}} \text { semestre (uniquement) } \\
2^{\mathrm{e}} \text { année }: 15 \text { heures par semestre }\end{array}$ \\
\hline PEL choisi & Cercles/Ranacles (PEL étudiant) \\
\hline Information des enseignants & $\begin{array}{l}\text { Au moyen de réunion, discussions, envois par } \\
\text { courriel et mise à disposition de documents }\end{array}$ \\
\hline Contraintes liées aux enseignants & $\begin{array}{l}\text { Recrutement tardif }{ }^{4} \text {, absence d'expérience du PEL, } \\
\text { voire absence d'expérience d'enseignement, } \\
\text { absence de motivation }\end{array}$ \\
\hline Information des étudiants & Réunion en amphithéâtre avant le début des TD \\
\hline Distribution et présentation du PEL & $\begin{array}{l}\text { En première semaine des TD du } 1^{\mathrm{e}} \text { semestre, par } \\
\text { les enseignants }\end{array}$ \\
\hline Discussion sur le PEL & $\begin{array}{l}\text { En troisième semaine au } 1^{\mathrm{e}} \text { semestre. Objectif : } \\
\text { aider les étudiants à remplir les rubriques du PEL }\end{array}$ \\
\hline Distribution des questionnaires & $\begin{array}{l}10^{\mathrm{e}} \text { semaine du } 1^{\mathrm{e}} \text { semestre, avant ou après le test } \\
\text { de fin de semestre, au choix des enseignants }\end{array}$ \\
\hline Nombre de questionnaires collectés & 116 \\
\hline $\begin{array}{l}\text { Réunion et nouvelle distribution de } \\
\text { questionnaires }\end{array}$ & $2^{\mathrm{e}}$ semestre \\
\hline $\begin{array}{l}\text { Nombre de questionnaires } \\
\text { supplémentaires obtenus après réunion de } \\
\text { début de second semestre }\end{array}$ & 42 \\
\hline
\end{tabular}



semestre, avant la reprise des TD, une réunion intitulée «Évaluation en anglais au premier et au second semestres ». Cette réunion était présentée comme obligatoire pour tous les étudiants inscrits en $1^{\mathrm{e}}$ et $2^{\mathrm{e}}$ années ayant choisi l'anglais. Elle visait à rappeler que le cursus d'anglais s'organisait sur trois ans, que le niveau attendu en fin de troisième année était B2, qu'il était important de s'auto-évaluer, que le PEL pouvait les y aider et qu'il leur permettrait aussi de planifier leur apprentissage. Sur les étudiants présents en début de réunion, vingt ont quitté l'amphithéâtre presque immédiatement, manifestant leur indifférence au PEL, et quarante-quatre de ceux qui n'avaient pas rempli le questionnaire au premier semestre ont accepté de répondre à nos questions.

\subsection{Le questionnaire} première question leur demande de cocher l'année d'étude ( $1^{\mathrm{e}}$ ou $2^{\mathrm{e}}$ année). Les deuxième et troisième questions visaient, d'une part, à éclaircir l'intérêt et l'implication des étudiants dans leur apprentissage des langues et, d'autre part, à relier le PEL à Dialang et à l'échelle européenne des niveaux, rappelant ainsi la cohérence de l'ensemble.

Le deuxième groupe de questions (questions 4 à 7) porte sur l'ergonomie du PEL et sa complémentarité avec les indications données en cours. En effet, la version Cercles/ Ranacles comporte une soixantaine de pages en tout, et après hésitation, nous avons choisi de toutes les distribuer aux étudiants, y compris le fascicule intitulé Annexe, qui décrit les niveaux sur une trentaine de pages. Ces trente pages ont pu nous sembler superflues, mais elles font pleinement partie du Portfolio de Cercles/Ranacles et nous savions que le Conseil de l'Europe ne valide les nouveaux PEL que lorsqu'ils sont accompagnés de listes de repérage très détaillées. D'ailleurs, la couverture de cette partie Annexe indique que «[...] les listes de repérage n'ont pas la prétention d'être exhaustives ». Par conséquent, nous avons décidé de les conserver telles quelles. En distribuant un fascicule si épais, l'idée sous-jacente était de vérifier si cette épaisseur desservait le propos du PEL. Les réponses devaient nous permettre de nous rendre compte si les enseignants avaient reçu sur le PEL une information suffisante pour être en mesure de répondre aux questions des étudiants ou s'il fallait renforcer cette information.

18 Les deux questions suivantes permettaient de vérifier comment les étudiants avaient organisé le temps passé à remplir le PEL, d'une part en début de semestre, et d'autre part éventuellement tout au long du semestre, pour, par exemple, se donner des objectifs et 
retourner cocher certaines cases lorsque l'objectif était rempli. Il s'agissait aussi de voir si les étudiants passaient plus de temps à essayer de se servir de leur PEL ou bien à travailler l'anglais en dehors du TD.

Les questions 10 à 12 et 14 s'intéressaient à la motivation. La question 10 portait sur la perception par les étudiants de la valorisation de leur multilinguisme, notamment des langues patrimoniales. La forte proportion, en Sciences économiques, d'étudiants d'origine étrangère (Afrique noire, pays de l'Est, Maghreb) est l'un des motifs qui nous avaient conduite à adopter le PEL à la rentrée 2003. Le multilinguisme, tel que l'entend le Conseil de l'Europe, recouvre la maîtrise, à des degrés divers, de plusieurs langues étrangères. Les questions 11,12 et 14 portaient sur les changements dans la manière d'apprendre induits par le PEL.

Les trois dernières questions sollicitaient l'appréciation globale des étudiants quant à leur intérêt pour le PEL (question 15) en terme d'efficacité à moyen terme (six mois à un an), d'utilité réelle (question 16, première partie), d'utilité perçue (question 16, seconde partie) et d'appropriation de l'outil (question 17).

21 Les résultats au questionnaire ont fait l'objet d'une saisie dans un logiciel de gestion de base de données.

\section{Résultats}

22 Nous avons collecté 158 questionnaires, dont $53,8 \%$ d'étudiants de $1^{\mathrm{e}}$ année et $46,2 \%$ de 2 e année. Les pourcentages indiqués ici et dans l'Annexe 2 sont arrondis au demi-point. La première observation est que tous les questionnaires n'ont pas été remplis dans leur totalité. Si plus de $85 \%$ ont répondu à toutes les questions du recto, moins d'un quart (24\%) ont répondu à l'ensemble de celles du verso. Un seul questionnaire comporte des réponses à chacune des 17 questions (hors questions portant sur l'année d'étude, et hors justifications). Cela ne peut s'expliquer parce que les étudiants n'ont pas lu le verso du questionnaire, puisque 92,6\% des étudiants ont proposé une réponse à au moins l'une des questions qui s'y trouvent. Le manque de temps pour répondre à des questions, dont beaucoup ne requéraient qu'une coche, suffit-il à expliquer la quasi-absence de questionnaires remplis totalement?

\subsection{Dialang}

23 La deuxième question a suscité un grand nombre de réponses, puisque deux étudiants seulement n'y ont pas répondu. Seuls $17,7 \%$ ont fait le test d'auto-évaluation Dialang, plus en $1^{\mathrm{e}}$ qu'en $2^{\mathrm{e}}$ année.

Quant aux raisons qui expliquent pourquoi ils n'ont pas utilisé Dialang, un sixième environ de ceux qui ne l'ont pas fait déclare de diverses manières ne pas en avoir eu le temps (14,5\% citent le mot «temps» dans leur réponse, formulée le plus souvent de la façon suivante: "pas le temps", «je n'ai pas eu le temps », etc. La deuxième raison à égalité est l'ignorance ; cela concerne 14,5\% étudiants. Pourtant, quatre diapositives de la présentation du PEL en début d'année concernaient Dialang. Les difficultés d'accéder à Internet depuis chez soi (3,8\% des réponses) sont aussi mentionnées, mais jamais celles pour y accéder depuis l'université. Ensuite, presque à égalité, les étudiants invoquent les problèmes techniques ( $6,8 \%$ des réponses), leur propre organisation (l'oubli est cité par 
$4,4 \%$ ) voire assurent qu'ils ont l'intention de faire ce test («je le ferai ce soir » ou « je le ferai demain » recueille $4,4 \%$ des réponses). La distinction par année n'est pas pertinente pour l'analyse des réponses à cette question.

\subsection{Le niveau cible}

Il était mesuré par les questions 3 et 13. Dans l'idéal, c'est le niveau $C$ que les étudiants aimeraient avoir, à 76,6\%, mais leur objectif est légèrement en dessous, puisqu'ils visent plutôt B2. Plus d'un étudiant de $2^{\mathrm{e}}$ année sur trois cible le niveau C2. Le fait que le niveau ciblé soit C pour $47,5 \%$ des étudiants confirme les résultats de Taillefer (2004), mais il semble que notre échantillon soit encore plus demandeur d'un excellent niveau en anglais. Doit-on y voir un effet de la mise en œuvre de la réforme dite du LMD ? Quant aux $3,1 \%$ qui ont répondu A1, ils se sont manifestement trompés sur la signification des indicateurs de niveaux, puisque à la question «Le PEL vous a-t-il permis de prendre conscience que vous en savez plus en langues que vous ne le pensez? », quatre sur cinq ont répondu oui pour l'anglais et trois ont même travaillé en dehors du cours grâce au PEL (question 14).

\subsection{L'ergonomie du PEL}

Elle est considérée comme globalement satisfaisante. Plus des deux tiers des étudiants ont trouvé que les objectifs du PEL y étaient clairement expliqués et seulement un quart ont jugé le PEL peu clair, mais bien expliqué par leur enseignant. Près de neuf étudiants sur dix ont compris les objectifs du PEL. Les enseignants, même sans aucune expérience antérieure du PEL, voire de l'enseignement, ont donc été clairs dans leur présentation aux étudiants.

Le PEL étudiant est considéré comme globalement ergonomique (première et seconde réponses à la question 5) pour plus de $92 \%$ des utilisateurs étudiants de premier cycle dans le cadre de l'enseignement présentiel. L'Annexe a été jugée claire et complète par $72,8 \%$, contre seulement $25,3 \%$ qui l'ont trouvée « difficile à comprendre et confus(e) ». Visiblement, l'épaisseur du document et son nombre élevé de pages ne le rendent pas rébarbatif pour plus des deux tiers des utilisateurs, mais près d'un tiers affirment tout de même avoir été gênés par cette partie.

Dans l'ensemble, le premier TD a été suffisant pour comprendre comment remplir le PEL et l'utiliser (pour près de $70 \%$ des étudiants). Un tiers tout de même avait toujours besoin de l'aide de leur professeur au-delà du premier TD, ce qui souligne bien l'importance d'un accompagnement des étudiants.

\subsection{Le PEL et la gestion du temps}

Cinquante-huit pour cent ont répondu qu'ils avaient mis « moins d'une heure » à remplir le PEL, et $29 \%$ « une à deux heures ». À peine $5 \%$ y ont passé plus de deux heures. Ces résultats nous ont étonnée à cause du nombre d'étudiants étrangers ou d'origine étrangère en $1^{\mathrm{e}}$ et $2^{\mathrm{e}}$ année $e^{5}$. Le PEL demande des efforts de réflexion, de synthèse, de rédaction, en particulier dans la partie Biographie, qui nécessitent bien plus d'une heure ${ }^{6}$. Vraisemblablement, les étudiants ayant répondu "moins d'une heure » et les douze étudiants qui n'ont pas répondu n'avaient pas, deux à quatre mois après la distribution du 
PEL, rempli toutes les rubriques de la Biographie. À noter que $5 \%$ ont tout de même passé plus de deux heures, un étudiant citant le chiffre de cinq heures et un autre celui de dix. Néanmoins, la plupart se sont contentés de cocher des cases, ce qui confirme l'impression de certains enseignants.

À peine 17 \% affirment utiliser le PEL régulièrement. Parmi les raisons invoquées par les non utilisateurs réguliers $(80 \%)$, celle qui revient le plus souvent est que le PEL leur semble inutile. Ce point de vue est exprimé de façon plus ou moins nuancée. La plupart ne rejettent pas le fait que le PEL puisse être utile. Simplement, ils ne voient pas à quoi il pourrait leur servir. Les mêmes nuances se retrouvent dans les autres réponses. Parmi les trois étudiants qui ont répondu ne pas l'utiliser régulièrement parce qu'il est mal fait, l'un tempère son propos en suggérant que le PEL est une «bonne idée ». Pour ce qui est des autres réponses, trois déclarent ne pas savoir travailler avec le PEL, huit ne pas y penser, deux ne pas avoir fait l'effort de s'en servir, deux considèrent qu'une auto-évaluation grâce au PEL est moins valide qu'une évaluation et deux estiment ne pas avoir été suffisamment incités à utiliser le PEL. Seuls 9,4\% invoquent le manque de temps. Il ressort de ces diverses réponses que le PEL ne leur semble pas avoir d'utilité immédiate par rapport à leurs enseignements reçus en TD. Ils ne retiennent des fonctions du PEL que celle d'auto-évaluation et s'en servent peu pour fixer des objectifs et travailler en dehors du TD. Il existe un décalage entre les objectifs assignés en TD et ceux qui proposés par le PEL, seuls les premiers étant identifiables et concrets.

\subsection{Le PEL et le multilinguisme}

La question 10 visait à vérifier si les étudiants percevaient la valorisation du multilinguisme opérée par le PEL et, au-delà, par notre université. La plupart des étudiants $(74 \%)$ se sont exprimés sur ce point et nous nous attendions à ce qu'ils citent des langues nombreuses et variées: celles étudiées au lycée, comme l'anglais ou l'allemand, des langues régionales, mais aussi l'arabe, l'hébreu et des langues africaines. Il n'en a rien été : seuls $16,5 \%$ se sont sentis valorisés, chiffre sans rapport avec la forte proportion d'étudiants étrangers inscrits en $1^{\mathrm{e}}$ et $2^{\mathrm{e}}$ années de Sciences économiques (environ un quart). La langue la plus citée, l'anglais, ne l'est que par 6,3\% des étudiants, et toutes les autres, c'est-à-dire l'allemand, l'espagnol, le portugais, l'arabe, le belge flamand, le tahitien et le français ne l'ont été qu'une fois ( $0,63 \%$ des étudiants à chaque fois). Aucune autre langue n'apparait, en particulier aucune langue parlée en Afrique autre que l'arabe. Comment se fait-il que le PEL ne remplisse pas cet objectif de valorisation, du multilinguisme pourtant explicitement formulé dans le PEL ? Dans ce cas, la maîtrise de langues "patrimoniales ${ }^{7}$ semble ne pas affecter la motivation, ce qui contredirait les observations de Clement, Dörnyei et Noels (1994: 277).

Le taux de réponses négatives à cette question (les trois-quarts) peut être mis en relation avec les 57 \% qui ont indiqué avoir passé moins d'une heure à remplir le PEL. Celui-ci a été distribué en TD d'anglais. Peut-être les étudiants s'attendaient-ils à ne faire l'objet d'un suivi du dispositif PEL par l'institution que pour l'anglais, les autres langues relevant pour eux de la sphère purement privée. 


\subsection{Le PEL et les objectifs d'apprentissage} concrets, plus en $2^{\mathrm{e}}$ qu'en $1^{\mathrm{e}}$ année. Une partie d'entre eux sont certainement ceux qui affirment l'utiliser régulièrement. Toutefois, rien n'indique que ces étudiants ont l'intention de travailler en autonomie pour réaliser leurs objectifs : peut-être comptentils surtout sur leur(s) professeur(s) pour atteindre ces objectifs. Le plus remarquable est la forte proportion ( $49 \%$ ) de ceux qui n'ont pas répondu à la question 11 . Ne l'ont-ils pas comprise? Doit-on y voir du désintérêt pour le PEL ou pour le questionnaire? N’ont-ils pas eu le temps de répondre? Par ailleurs, ceux qui répondent ne pas s'être fixés de nouveaux objectifs sont près d'un quart. Pourquoi? Deux raisons sont invoquées: certains déclarent être autonomes et ne pas avoir besoin du PEL pour se fixer des objectifs ; d'autres estiment, de façon très peu explicite, que les objectifs du PEL ne sont pas suffisamment concrets ou, en tous cas, ne les concernent pas (réponses du type « utiliser le PEL c'est ennuyeux » ou « je n'en vois pas l'intérêt »). Concernant les langues citées, celle qui revient le plus est l'anglais, dans près de $80 \%$ des cas, mais l'ont été aussi le turc et l'hébreu, langues qui n'apparaissaient pas dans les réponses à la question 10 . Quatre étudiants ont cité deux langues en plus du français et deux en ont cité trois. Par conséquent, l'interprétation des réponses à cette question dépend de l'observateur. On peut soit déplorer que le PEL n'ait profité qu'à un quart des étudiants, soit constater que le PEL a tout de même permis de donner de nouveaux objectifs d'apprentissage à $27,2 \%$ des étudiants. Quant à savoir ce qu'il faut faire pour les aider à se fixer de nouveaux objectifs d'apprentissage, toutes les réponses suggèrent d'augmenter l'exposition aux langues.

Bien que $27,2 \%$ se soient fixé des objectifs grâce au PEL, 48 \% s'y sont tenus ou s'y tiendront. Cette contradiction s'explique sans doute parce qu'ils ont compris la question, formulée ainsi: "Vous êtes vous tenus, vous tenez-vous ou vous tiendrez-vous à ces objectifs? », comme "vous tiendrez-vous aux objectifs énoncés dans le PEL », et non «vous tiendrez-vous à des objectifs que le PEL vous aura permis de formuler?». La plupart ont mal interprété l'anaphorique «ces». Aussi le « oui » à la question 12 doit-il être compris plutôt comme un « oui, j'ai effectué du travail personnel en dehors du TD ». Certains ne semblent pas différencier travail autonome et travail personnel. Cette interprétation est confirmée par les raisons des tenants du «non » à la question $12:$ : les cours de langues ne sont pas assez structurés » et "on n'a qu'une heure et demie d'anglais par semaine ; c'est insuffisant ». D'autres invoquent la difficulté de travailler en autonomie et le manque de motivation.

Si 63 \% n'ont pas changé leurs habitudes de travail grâce au PEL, il n'en reste pas moins que 27 \% l'ont fait de diverses façons (travail en centre de langues, discussions avec amis étrangers, travail personnel à l'aide de manuels, etc.). Toutefois, de tels comportements, très concrets, demeurent assez peu motivants dans l'échelle de la motivation, comme le souligne Toffoli (2003). En outre, ils ne précisent pas ce qu'ils ont appris au cours de ce travail. 


\subsection{L'appropriation du PEL par les étudiants}

36 À notre grande surprise, malgré la difficulté à travailler régulièrement avec le PEL et la difficulté, plus généralement, à étudier en autonomie, $36,5 \%$, plus en $1^{\mathrm{e}}$ année qu'en $2^{\mathrm{e}}$, déclarent avoir l'intention de l'utiliser ultérieurement, même si leur enseignant ne le leur demande pas. Cette proportion est légèrement inférieure à celle des étudiants de $1^{\mathrm{e}}$ année qui validera sans doute ses deux semestres, environ la moitié (les inscrits en anglais en $2^{\mathrm{e}}$ année représentent $49 \%$ de ceux de $1^{\mathrm{e}}$ année). On peut s'interroger cependant sur la manière dont ils l'utiliseront : pour s'évaluer ou pour apprendre?

Toutefois, lorsque l'on demande s'ils trouvent le PEL motivant, près de $60 \%$, répondent par la négative, mais $34 \%$ de ceux qui sont en DEUG $2^{\mathrm{e}}$ année répondent « oui ». Là encore, le fait de considérer ces $34 \%$ comme encourageants ou non dépend du point de vue de l'observateur. Les $27,1 \%$ des $1^{\mathrm{e}}$ année qui ont répondu par l'affirmative sont-ils aussi ceux, presque deux fois plus nombreux ${ }^{8}$, qui valideront leur $1^{\mathrm{e}}$ année ? Ces chiffres indiquent en tous cas que l'un des deux objectifs du PEL semble ne pas être rempli : le PEL n'augmente pas significativement la motivation. Il reste qu'un tiers des étudiants le trouvent tout de même « motivant ".

En revanche, et c'est là une des multiples surprises que nous ont réservé les réponses à ce questionnaire, $55 \%$ des 158 interrogés jugent le PEL «intéressant», à proportion équivalente en $1^{\mathrm{e}}$ et $2^{\mathrm{e}}$ année. Ils distinguent nettement efficacité et intérêt. Ceci confirme la distinction établie par Crookes et Schmidt (cités par Dörnyei 1994 : 277) entre « interest ", c'est-à-dire la curiosité individuelle et le désir d'en savoir plus sur un sujet, et « relevance ", qui est le besoin d'en connaître davantage pour des raisons de valeurs et d'objectifs personnels.

\section{Conclusion}

Cette étude présente des défauts. Le questionnaire, par exemple, n'a pas incorporé les modèles théoriques permettant de décrire en particulier l'ergonomie, le travail en autonomie ou la motivation. Il n'a pas éclairci certains concepts pour les étudiants qui, manifestement, auraient pu être davantage guidés dans leurs réponses. Il suscite des questions sur l'interprétation de certaines données.

Pourtant, il nous permet de dresser un portrait de l'étudiant-type qui y a répondu. Cet étudiant-type n'a pas fait le test en ligne Dialang. Cependant, il a bien compris l'échelle européenne des niveaux. Dans l'idéal, il aimerait avoir le niveau le meilleur (C2) et il vise un niveau à peine inférieur. Il trouve les objectifs du PEL clairement expliqués et le PEL lui paraît compréhensible, bien qu'organisé de façon complexe. Le descriptif des niveaux lui semble clair et complet. Les explications de la première séance de TD lui ont suffi pour remplir le PEL chez lui en moins d'une heure, même s'il n'a fait que survoler certaines rubriques sans rédiger. Il n'utilise pas le PEL régulièrement pour de multiples raisons : d'abord, travailler l'anglais en TD lui paraît d'utilité plus immédiate. D'ailleurs, il ne se sent pas régulièrement incité à utiliser le PEL, et par conséquent ne s'en sert ni régulièrement ni activement. Il lui réserve plutôt un rôle subsidiaire. De toute façon, il ne pense pas que la réflexion en français sur sa manière d'apprendre lui permettra de progresser en anglais. Il juge le Portfolio intéressant, mais pas motivant. Nous n'avons retenu ici que les réponses aux questions sur lesquelles se sont exprimés plus de la moitié 
de ceux qui ont rempli le questionnaire. On peut le constater, le bilan de l'introduction du PEL en enseignement présentiel en $1^{\mathrm{e}}$ et $2^{\mathrm{e}}$ années de Sciences économiques reste nuancé. Le PEL utilisé avec un encadrement minimal n'a pas eu tous les effets escomptés. Il n'a pas permis à la plupart de ceux qui possèdent à des degrés divers des langues qui ne bénéficient pas d'une reconnaissance académique de se sentir valorisés. La majorité des étudiants est consciente de l'importance de la maîtrise de l'anglais à un niveau élevé, mais pas de l'importance du multilinguisme, voire peu convaincue d'un multilinguisme pourtant réel.

41 En revanche, les étudiants n'ont pas non plus massivement rejeté le PEL comme inutile et chronophage. Au contraire, plus de la moitié l'apprécient. Un quart déclarent posséder ou avoir développé, grâce au PEL, des aptitudes à l'étude et des aptitudes heuristiques suffisantes pour atteindre les objectifs qu'ils se sont fixés grâce au PEL. Plus d'un tiers disent compter l'utiliser en l'absence de guidage de l'enseignant dans le semestre ou l'année qui vient. Un tiers le jugent motivant, plus de la moitié intéressant et ils se le sont appropriés au point d'envisager de le recommander. Par conséquent, le PEL modifie effectivement la représentation qu'une minorité non négligeable d'étudiants se fait de son apprentissage.

Il est frappant que ce soit précisément la partie à rédiger de la Biographie langagière portant sur la réflexion sur son apprentissage des langues, et au même type de questions du questionnaire (questions 10 à 14), qui aient été remplies de la façon la moins complète. Or, la Biographie est un élément crucial du PEL. Peut-on faire en sorte que les étudiants ne rejettent pas les tâches métacognitives? Il existe d'autres utilisations possibles du PEL, moins centrées sur la Biographie et davantage sur la partie Dossier. Une recherche future pourra se baser sur les éléments permettant de définir la motivation ${ }^{9}$, des étudiants bien entendu, mais aussi des enseignants, pour voir de quelle façon le PEL peut l'augmenter, si cela est possible. Cette recherche pourrait aussi nous permettre de construire des questionnaires en vue de produire un PEL peut-être plus motivant, compte tenu des caractéristiques de nos étudiants et des contraintes locales. Il faut aussi envisager d'augmenter la motivation des enseignants à l'utiliser et transformer en motivation l'intérêt que suscite le PEL parmi les étudiants.

\section{BIBLIOGRAPHIE}

Clement, Richard, Zoltán Dörnyei et A. Kimberly. Noels. 1994. « Motivation, self-confidence and group cohesion in the foreign language classroom ». Language Learning 44, 417-448.

Deci, Edward L. et Richard M. Ryan. 1994. « Promoting self-determinated education ». Scandinavian Journal of Educational Research 38/1, 3-14.

Conseil de l'Europe. 2000. Cadre Européen Commun de Référence pour les Langues : apprendre, enseigner, évaluer. Paris, Strasbourg : Conseil de l'Europe / Didier. 
Division des politiques linguistiques de Strasbourg. 2004 [2000]. Portfolio européen des langues : principes et lignes directrices. <http://culture2.coe.int/portfolio/inc.asp?L=F\&M=\$t/208-1-0-1/ main_pages/documentsf.html>.

Kohonen, Viljo. 2001. « Exploring the educational possibilities of the 'Dossier': Suggestions for developing the pedagogical function of the European Language Portfolio ». In Division des politiques linguistiques de Strasbourg, Enhancing the pedagogical aspects of the European Language Portfolio (ELP), 2-30. 28 juillet 2005 <http://commonweb.unifr.ch/cerle/pub/cerleweb/portfolio/ fondement/documentation/>.

Dörnyei, Zoltán. 1994. « Motivation and motivating in the foreign language classroom ». The Modern Language Journal 78/3, 273-284.

Dörnyei, Zoltán. 2005. « The internal structure of language learning motivation and its relationship with language choice and learning effort ». The Modern Language Journal 89/1, 19-36.

Gardner, Robert et Peter D. MacIntyre. 1993. « A student's contributions to second-language learning. Part II: Affective variables ». Language Teaching 26, 1-11.

Little, David. 2000. Exploitation du Portfolio européen des langues : neuf exemples. Strasbourg : Conseil de l'Europe. <http://culture2.coe.int/portfolio//documents/Exploitation \%20du \% 20PEL.pdf>.

Little, David et Radka Perclová. 2001. Guide à l'usage des enseignants et formateurs d'enseignants. Strasbourg : Conseil de l'Europe. <http://culture2.coe.int/portfolio//documents/ ELPguide_teacherstrainersf.pdf>.

Schärer, Rolf. 2000. Rapport final Portfolio Européen des Langues Phase pilote 1998-2000. Strasbourg : Conseil de l'Europe. <http://culture2.coe.int/portfolio/inc.asp?L=F\&M=\$t/208-1-0-1/ main_pages/../\&L=F\&M=\$t/208-1-0-1/main_pages/documentsf.html>.

Schärer, Rolf. 2004. Portfolio Européen des Langues. De l'expérimentation à la mise en œuvre (2001 - 2004) - Rapport de synthèse - Version finale <http://culture2.coe.int/portfolio/inc.asp?L=F\&M= \$t/208-1-0-1/main_pages/../\&L=F\&M=\$t/208-1-0-1/main_pages/documentsf.html>.

Schneider, Günther et Peter Lenz. 2001. Portfolio européen des langues : Guide à l'usage des concepteurs. Strasbourg : Conseil de l'Europe. <http://culture2.coe.int/portfolio// documents_intro/Fguide.pdf>.

Taillefer, Gail. 2004. « Une analyse critériée des besoins linguistiques dans l'enseignement universitaire de Sciences économiques ». ASp 43-44, 107-124.

Toffoli, Denyze. 2003. « De la théorie à la pratique : appliquer des modèles cognitifs de la motivation dans un centre de langues ». ASp 41-42, 99-114.

Vallerand, Robert J. et Céline Blanchard. 1998. «Éducation permanente et motivation : contribution du modèle hiérarchique de la motivation intrinsèque et extrinsèque ». Éducation permanente $136 / 3,15-36$.

\section{ANNEXES}

Annexe 1 


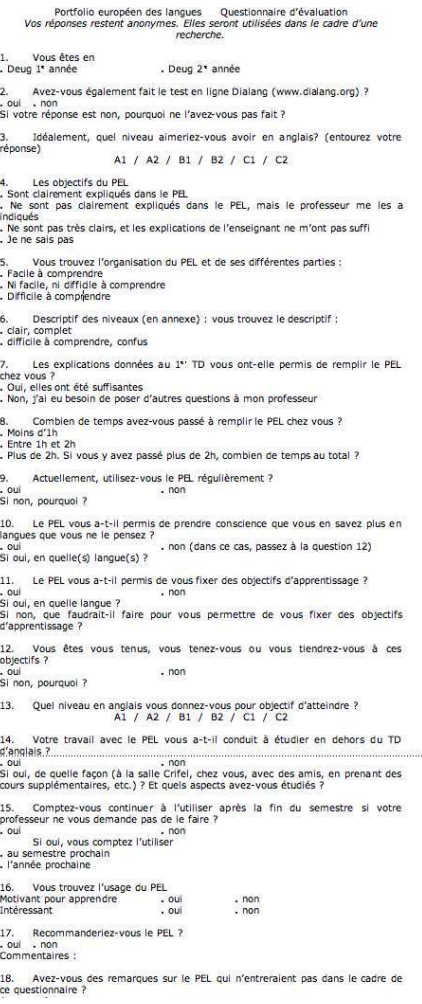

\section{Annexe 2}

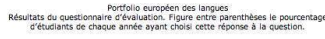

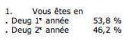

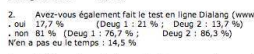

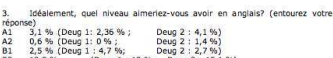

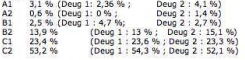

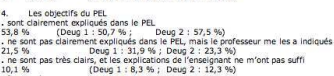

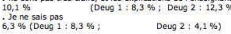

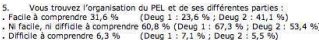

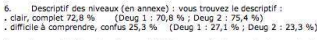

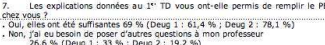

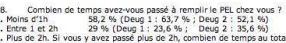

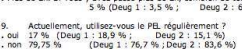

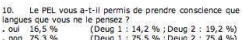

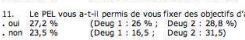

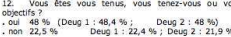

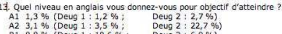

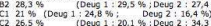

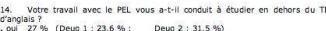

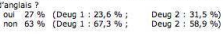

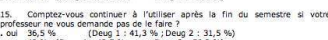

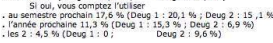

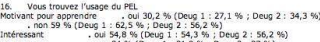

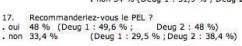

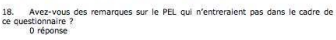




\section{NOTES}

1. < http://culture2.coe.int/portfolio/inc.asp?L=F\&M=\$t/208-1-0-1/main_pages/../\&L=F\&M=\$t/ 208-1-0-1/main_pages/introductionf.html>, site consulté le 21/06/2005.

2. <http://www.alte.org/projects/eelp.cfm>.

3. Ce test était gratuit en 2004-2005. Sa conception a bénéficié du financement de la Commission européenne dans le cadre du programme Socrates Lingua action D.

4. Les trois non-titulaires intervenant en cycle de Licence ont été recrutés entre mi-septembre et mi-octobre. Sur cinq enseignants, deux étaient titulaires. Une personne n'avait aucune expérience de l'enseignement.

5. Cent quarante-quatre étudiants étrangers étaient inscrits en $1^{\mathrm{e}}$ année de Sciences économiques en 2004-2005 et quatre-vingt quatre en $2^{\mathrm{e}}$ année, soit environ un quart des étudiants.

6. Les cinq rubriques suivantes de la Biographie demandaient à être rédigées : « Les expériences d'apprentissage de langues auxquelles j'accorde une grande importance et/ou qui m'ont beaucoup marqué(e) », "Comment j'ai participé aux aspects culturels associés à la/aux langue(s) seconde(s)/étrangère(s) que je connais ", "Langues patrimoniales ", " Mon prochain objectif d'apprentissage est le suivant » et " Apprendre à apprendre ».

7. Les langues «patrimoniales » sont définies par le PEL comme « la/les langue(s) des immigrants qui arrivent dans un nouveau pays, les langues des minorités ethniques, les langues utilisées dans le contexte des célébrations religieuses ou de festivals culturels ".

8. Le nombre d'étudiants ayant choisi l'anglais en $2^{\mathrm{e}}$ année représentait à la rentrée $49,5 \%$ de celui de $1^{\mathrm{e}}$ année en 2004-2005.

9. Notamment les travaux de Vallerand (1993), Deci \& Ryan (1994), Vallerand \& Blanchard (1998) et Dörnyei (2005).

\section{RÉSUMÉS}

Le Conseil de l'Europe et les enseignants qui utilisent le Portfolio européen des langues (PEL) avec leurs étudiants font le pari que cet instrument favorisera l'autonomie et accroîtra la motivation. Quel est le point de vue des utilisateurs étudiants? Cette étude se propose de l'examiner au moyen d'un questionnaire portant sur l'ergonomie du PEL, les changements qu'il induit dans leur apprentissage par son utilisation en semi-autonomie, la valorisation de leur multilinguisme, leur intérêt pour le PEL et les effets du PEL sur leur motivation. Le questionnaire a été administré en 2004-2005 à 158 étudiants de cycle de Licence de Sciences économiques ( $1^{\mathrm{e}}$ et $2^{\mathrm{e}}$ années). Son analyse suggère une réaction mitigée des étudiants face au PEL, plutôt favorables à son introduction, mais considérant qu'il reste globalement sans effet sur leur motivation.

The Council of Europe and teachers using the European Language Portfolio (ELP) with their students hope that it will be a tool to favour autonomy and increase motivation. What is the student users' point of view? Data collected from a questionnaire administered in 2004-2005 to 158 first- and second-year Economics students was used to look at ELP ergonomics, the changes induced by their semi-autonomous use of the ELP, learners' perception of their own 
multilingualism and their interest in the ELP as well as any effects of the ELP on motivation. Mixed responses from the students would suggest that they are initially, at least, favourable to using the ELP, though it appears to have little effect on overall motivation.

INDEX

Mots-clés : ergonomie, motivation, multilinguisme, PEL, portfolio européen des langues

Keywords : ELP, ergonomics, European language portfolio, motivation, multilingualism

\section{AUTEUR}

\section{ANNE-MARIE BARRAULT-MÉTHY}

Anne-Marie Méthy est maître de conférences à l'Université Montesquieu-Bordeaux 4 depuis la rentrée 2001 et membre de la FRE 2791. Elle coordonne les enseignements d'anglais pour le cycle de Licence de Sciences économiques. ammethy@u-bordeaux4.fr 